\title{
Effect of Chamber Backpressure on Swirl Injector Fluid Mechanics
}

2008 Joint Propulsion Conference

\author{
R. Jeremy Kenny \\ James R. Hulka \\ Marlow D. Moser \\ Noah O. Rhys
}




\section{Outline}

- Background

- Objective

- Results

- Conclusions/Discussion 


\section{Background}

- A common propellant combination used for high thrust generation is GH2/LOX.

- Historical GH2/LOX injection elements have been of the shear-coaxial type.

- Element type has a large heritage of research work to aid in element design.

- The swirl-coaxial element, despite its many performance benefits, has a relatively small amount of historical, LRE-oriented work to draw from.

- Design features of interest are grounded in the fluid mechanics of the liquid swirl process itself, are based on data from low-pressure, low mass flow rate experiments.

-There is a need to investigate how high ambient pressures and mass flow rates influence internal and external swirl features.
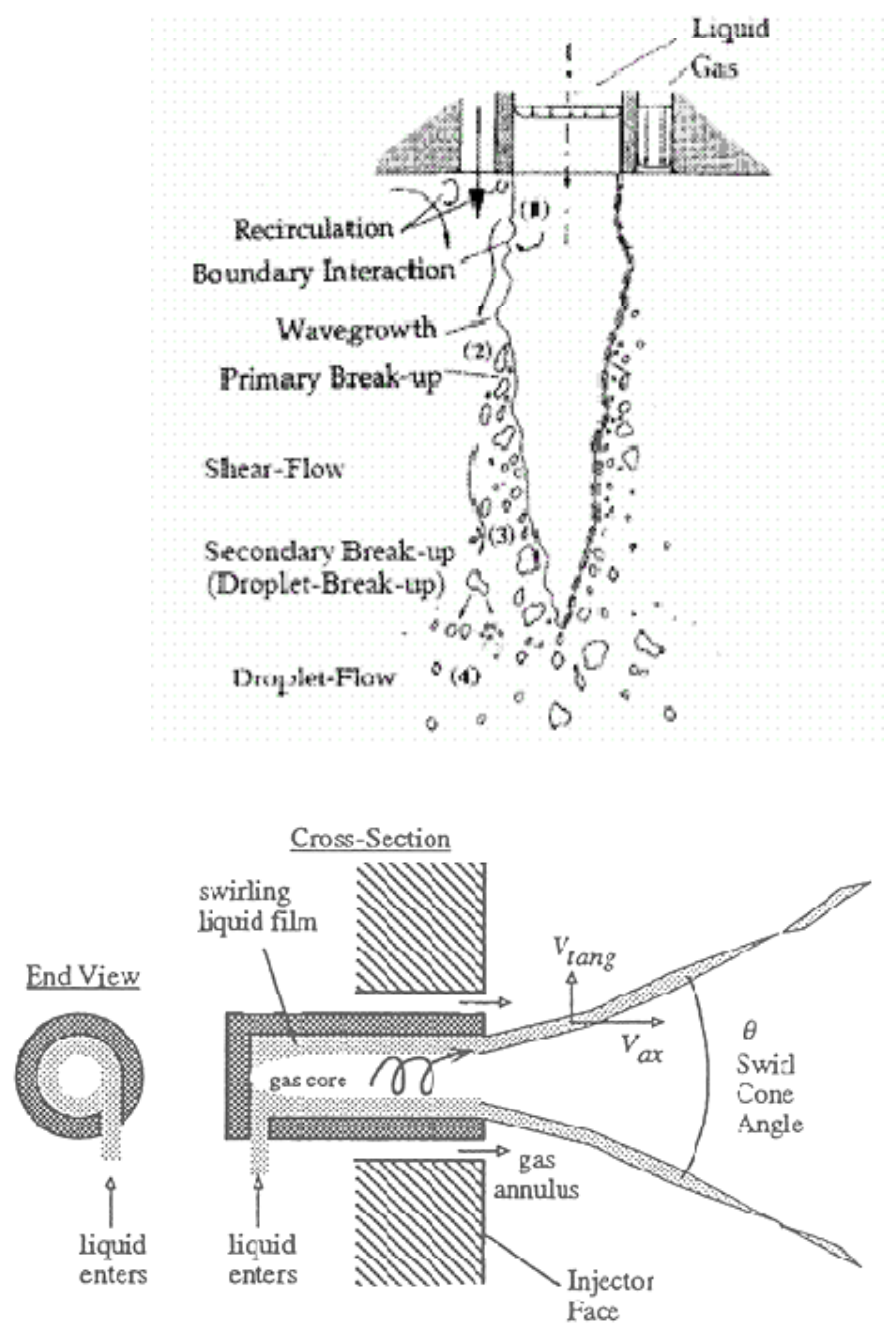


\section{Objective}

- Determine influence of varying liquid mass flow rate and ambient chamber pressure on the intact-length fluid mechanics of a liquid swirl element

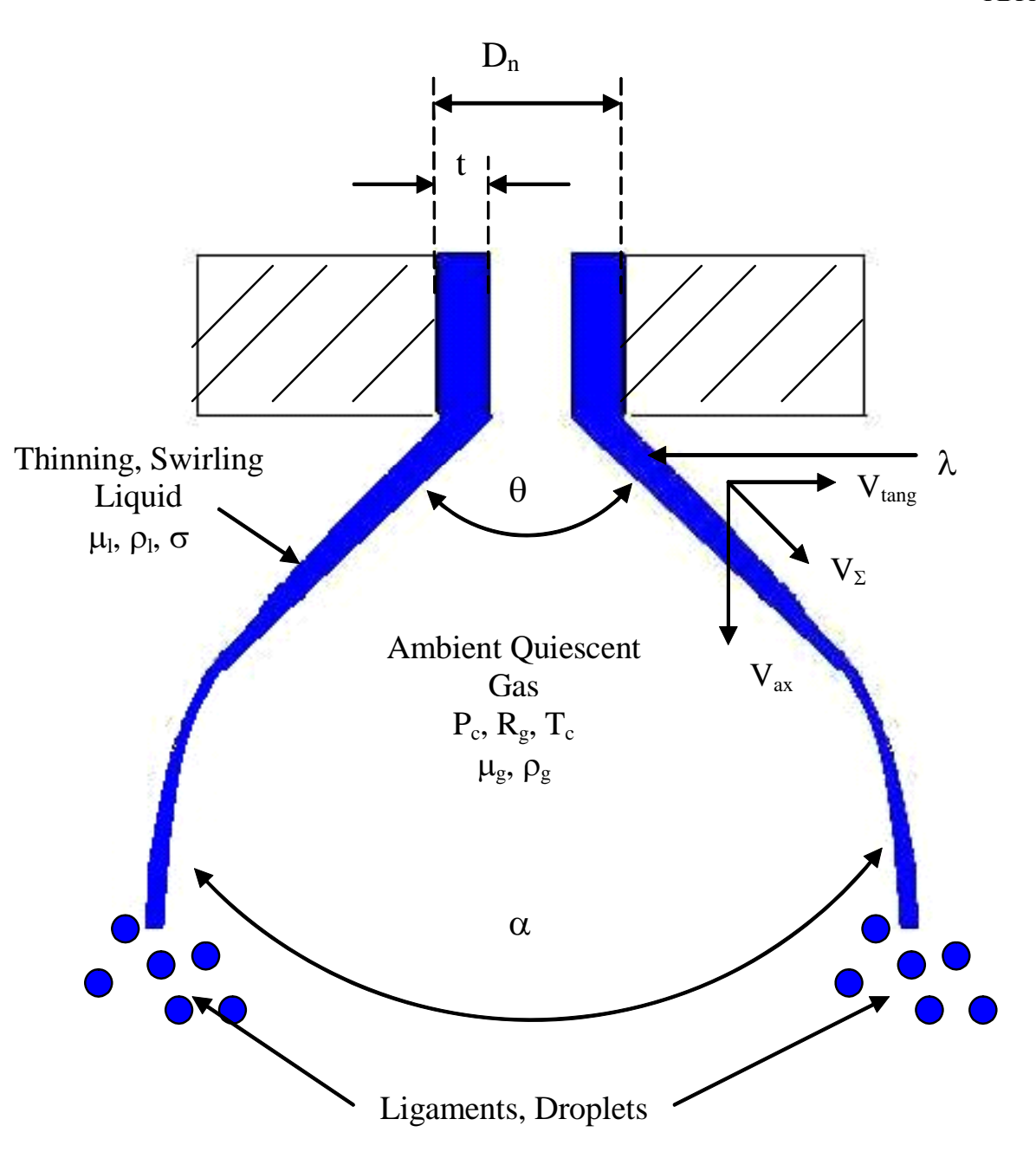




\section{Cold Flow Facility}

- Water/Nitrogen Injector Spray Test Rig (WNIST)

- Simulates LOX/gaseous fuel by H2O/GN2

- Ambient chamber pressure set up to 1400 psia by additional GN2 feed lines

$-\mathrm{H} 2 \mathrm{O}$ mass flow rates up to $1 \mathrm{lbm} / \mathrm{s}$

- Real-time controllable backpressure, flow rates, and gas temperature 


\section{Cold Flow Facility}
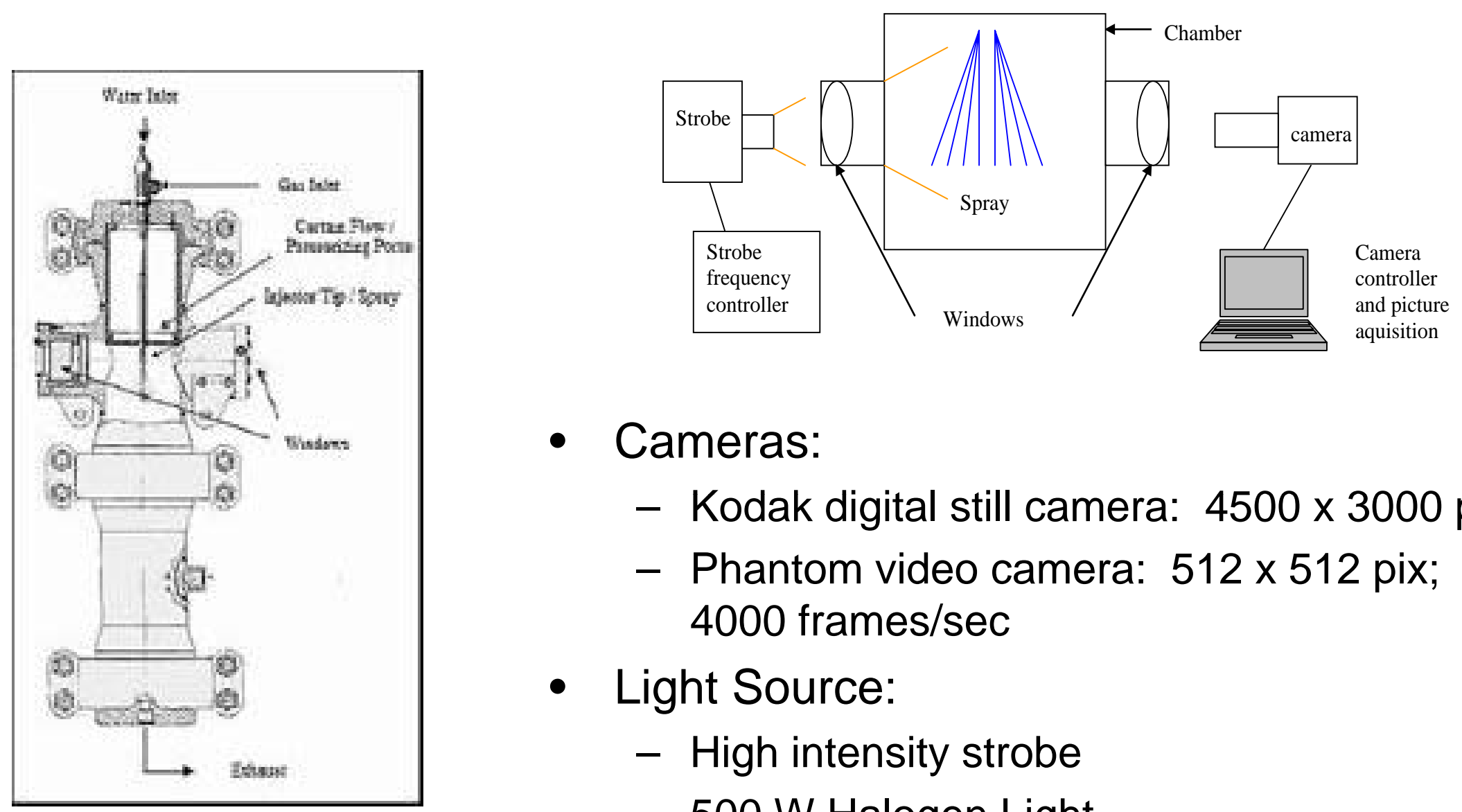

- Cameras:

- Kodak digital still camera: 4500 x 3000 pix

- Phantom video camera: 512 x 512 pix; 4000 frames/sec

- Light Source:

- High intensity strobe

- 500 W Halogen Light 


\section{Diagnostic Methods}

- Metering of:

- Upstream liquid static pressure

- Chamber pressure

- Liquid mass flow rates

- Fluid temperatures

- Spray Profile through Shadowgraph Imaging

- Inner film thickness profile

- External spray boundary and cone angle 


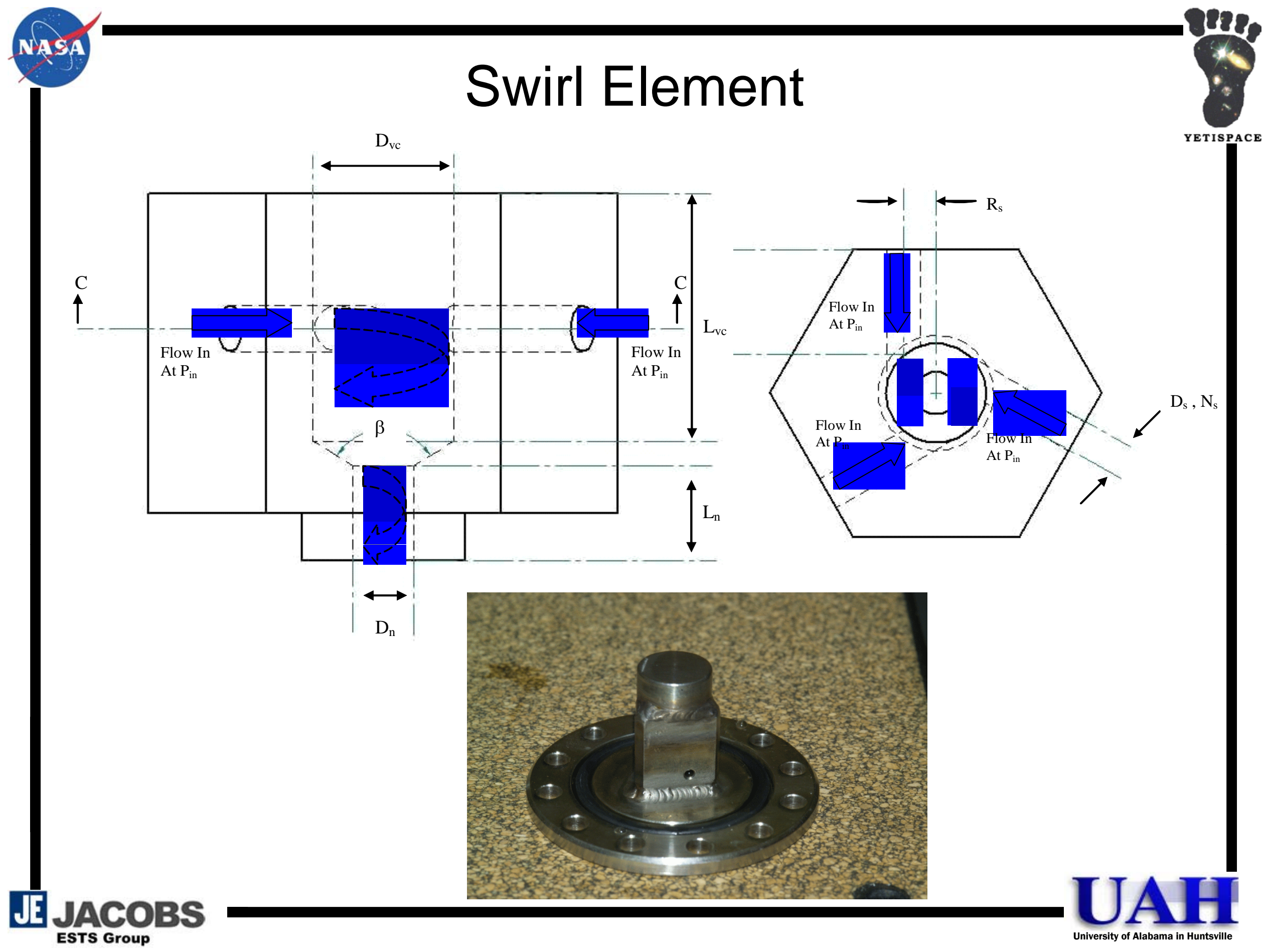




\section{Swirl Element Cont.}

- Inner flow structure seen by clear acrylic section

- Similar acrylic section used with squared bottom for external spray features
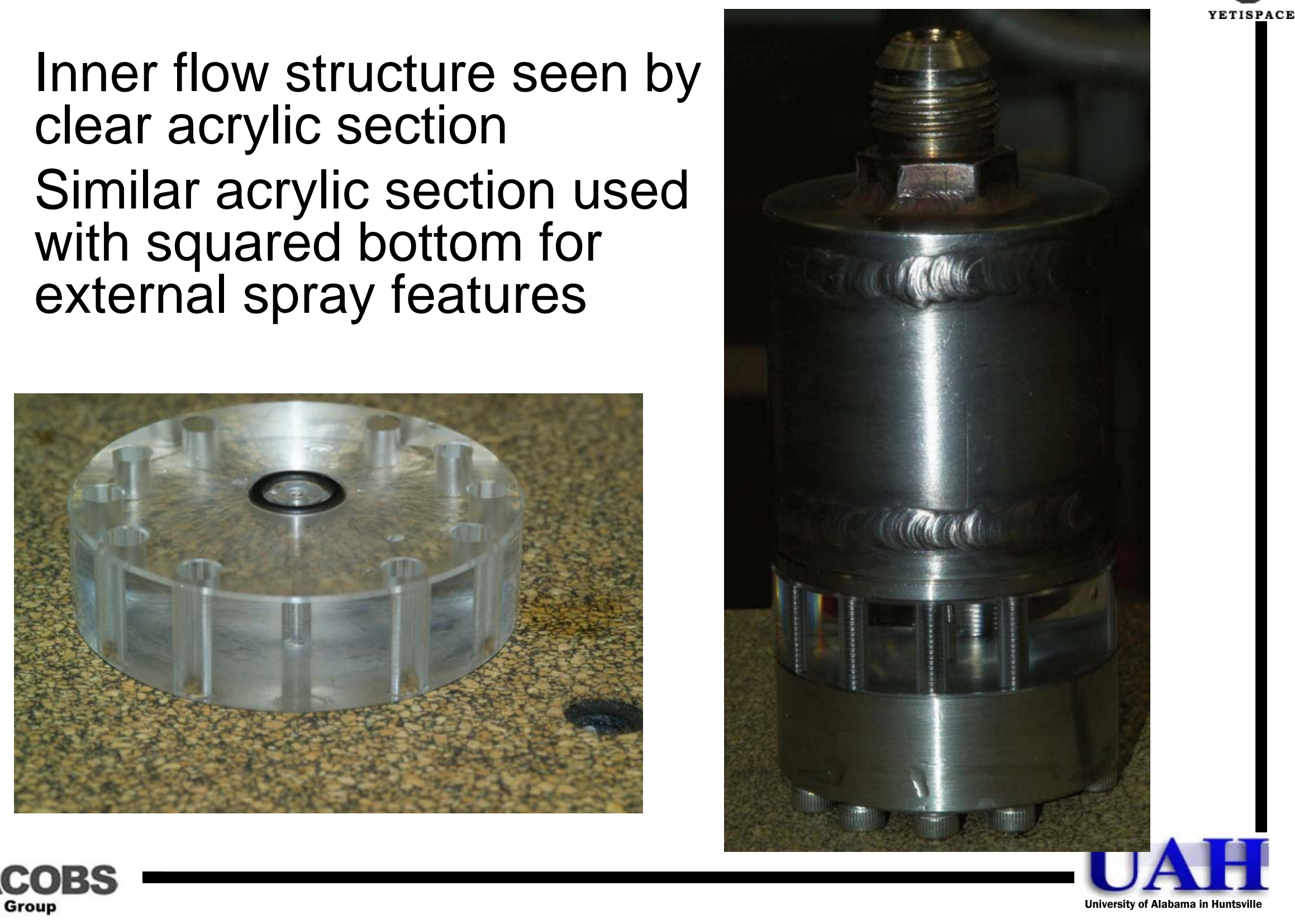


\section{Design Methodology}

- Doumas \& Laster gives relations between swirl features via experimental work

- Incorporates friction effects

- No chamber pressure influences

- No information about off-design mass flow rate operation

- Bazarov gives relations between swirl features via analytical approach

- Can incorporate friction effects

- No ambient pressure influences 


\section{Swirl Element Design Parameters}

\begin{tabular}{|c|c|c|}
\hline Parameter & Bazarov Prediction & Doumas \& Laster Prediction \\
\hline Mass Flow Rate (kg/s) & 0.09 & 0.09 \\
\hline Free Cone Spray Angle (deg) & 49 & 2.09 \\
\hline Pressure Drop at Design Flow (MPa) & 1.72 & 0.414 \\
\hline Discharge Coefficient & 0.463 & 0.40 \\
\hline Film Thickness (mm) & 0.43 & 1.58 \\
\hline Orifice Diameter (mm) & 1.58 & 1.55 \\
\hline Orifice to Centerline Radius (mm) & 1.55 & 3.35 \\
\hline Vortex Chamber Diameter (mm) & 3.35 & N/A \\
\hline Orifice Length (mm) & 3.73 & N/A \\
\hline Vortex Chamber length (mm) & 3.91 & 2.08 \\
\hline Nozzle Diameter (mm) & 2.08 & N/A \\
\hline Nozzle Length (mm) & 16.05 & \\
\hline
\end{tabular}




\section{Inner Film Thickness Videography}

- Show movies of internal flow at full flow rate and chamber pressure of:

- 0.10 MPa

$-0.69 \mathrm{MPa}$

- $1.03 \mathrm{MPa}$

- $1.38 \mathrm{MPa}$

$-1.72 \mathrm{MPa}$

$-2.07 \mathrm{MPa}$

$-2.76 \mathrm{MPa}$

$-4.83 \mathrm{MPa}$ 


\section{Inner Film Thickness Profiles}
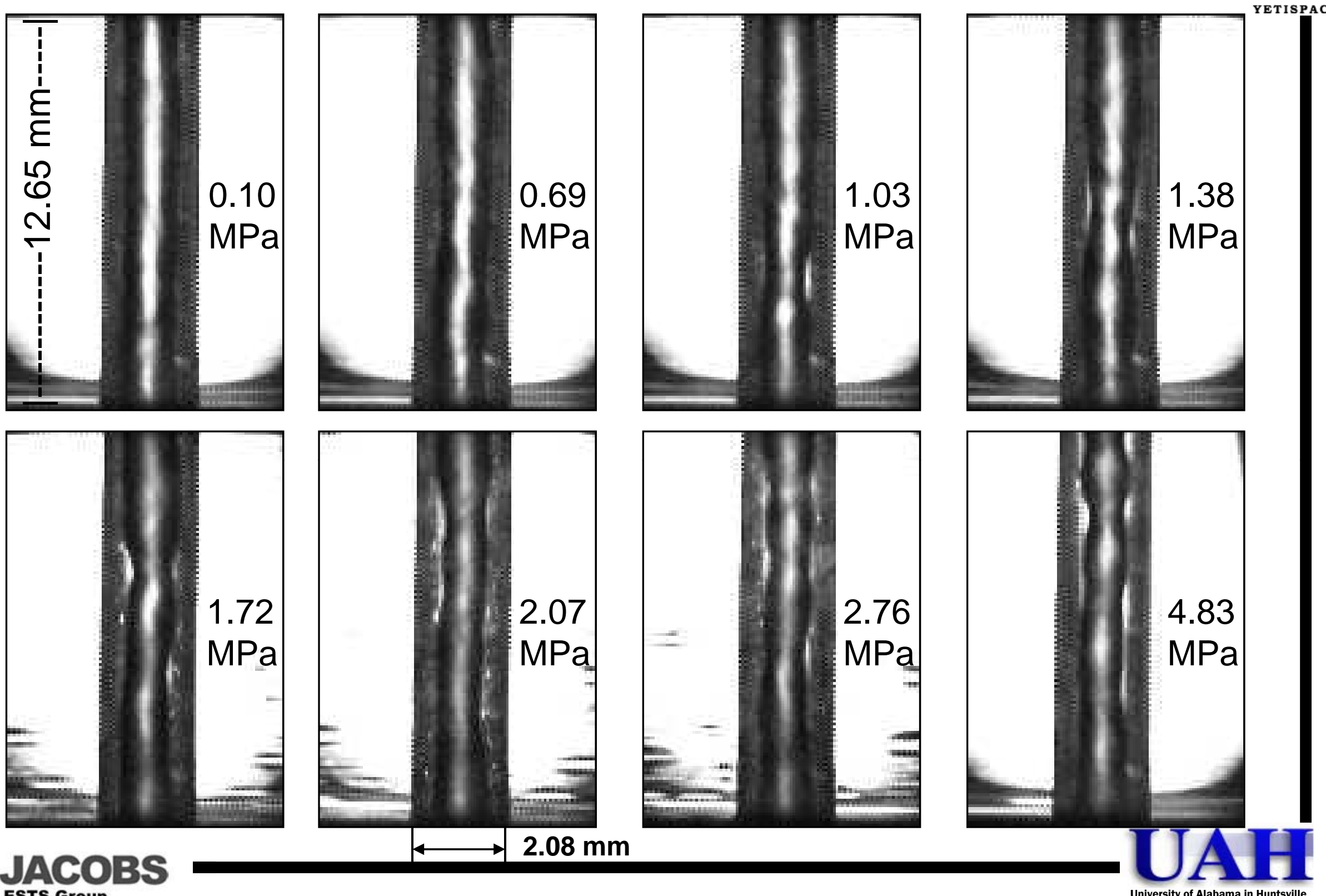


\section{Spatial Film Thickness}

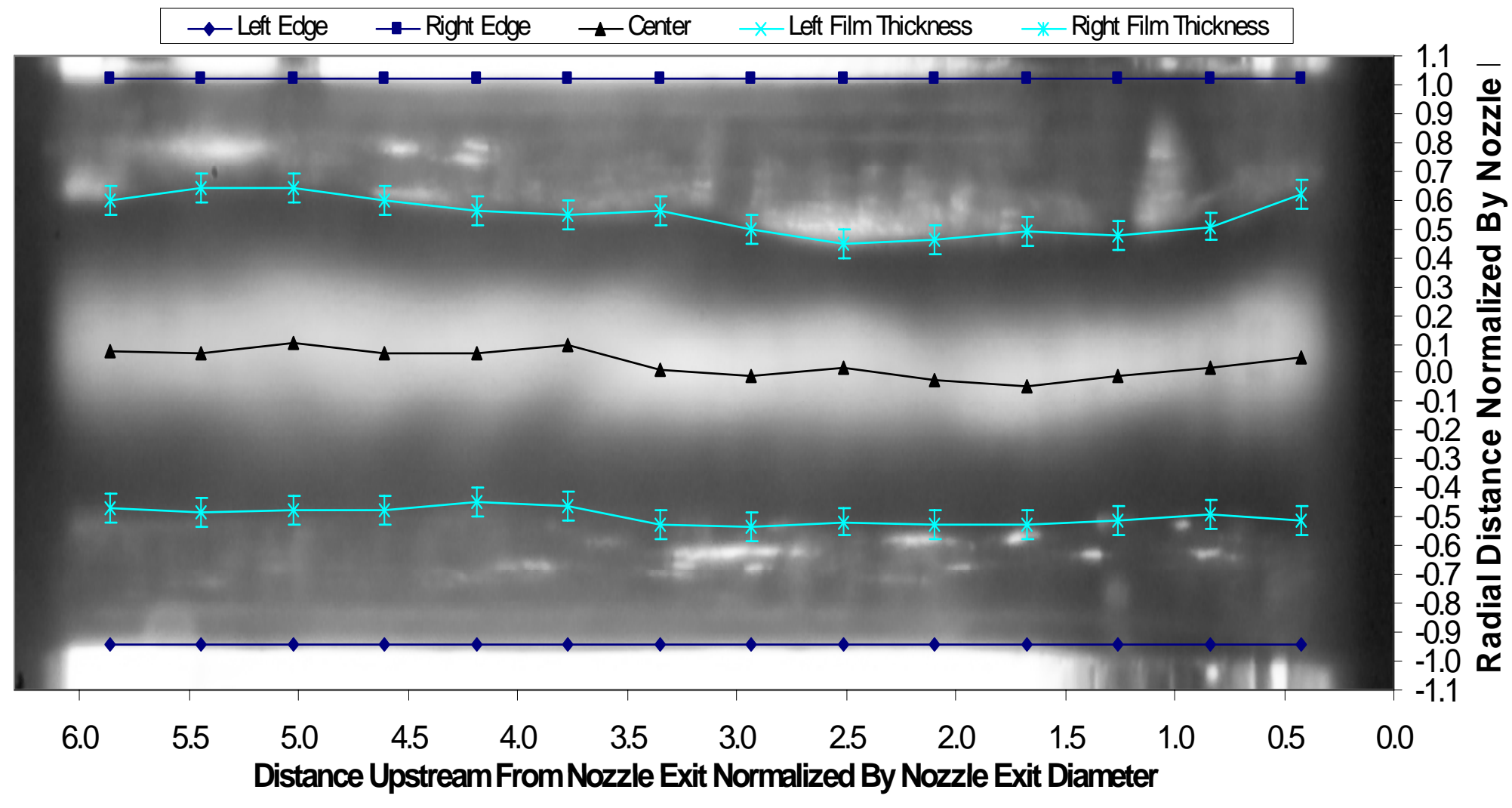

- Corrected for optical effects

- Both left and right sides profiles measured and used to find average film thickness profile 


\section{Inner Film Thickness Quantification}

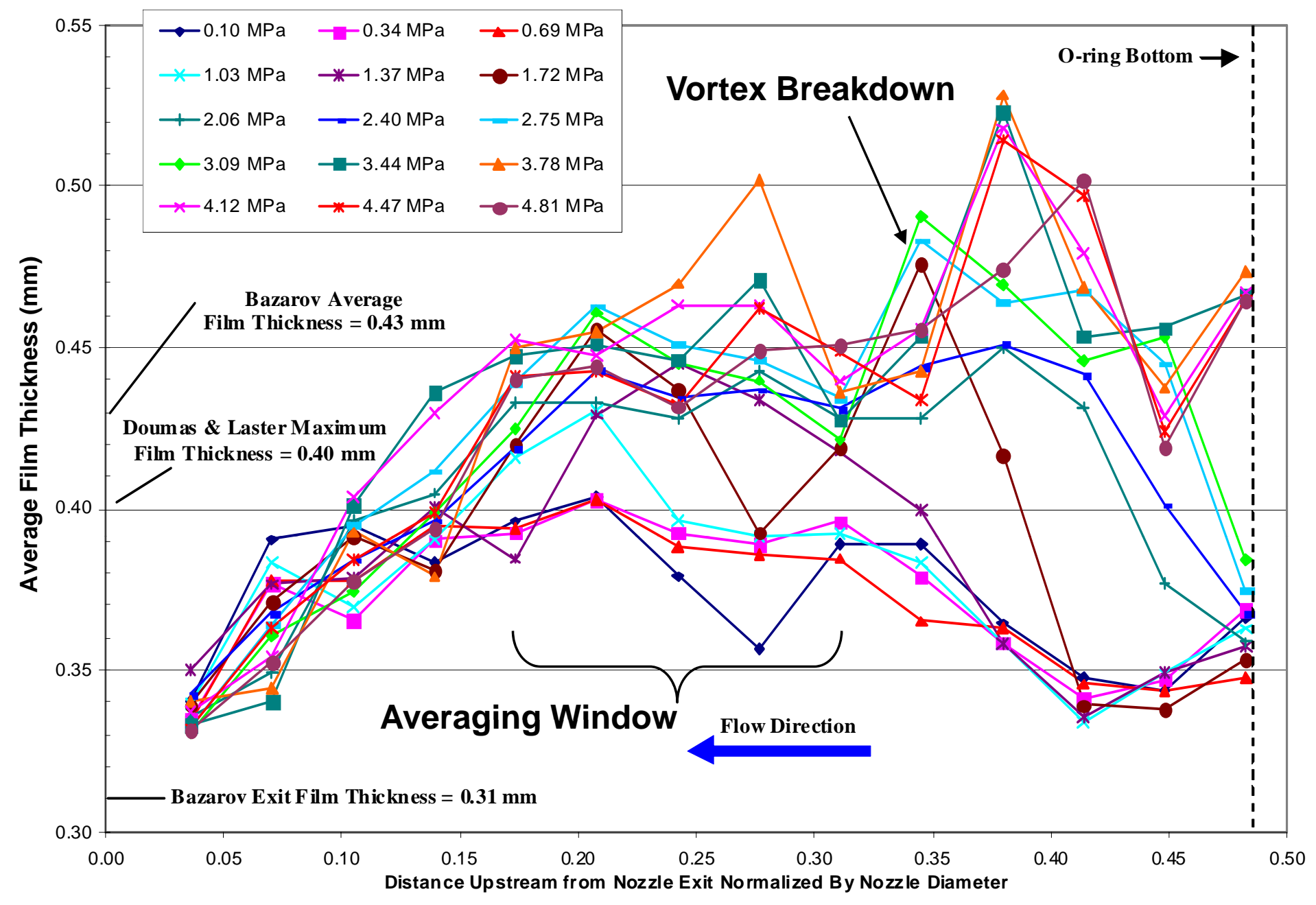

- Fixed design mass flow rate, varying chamber pressures. 


\section{Average Film Thickness Variation}

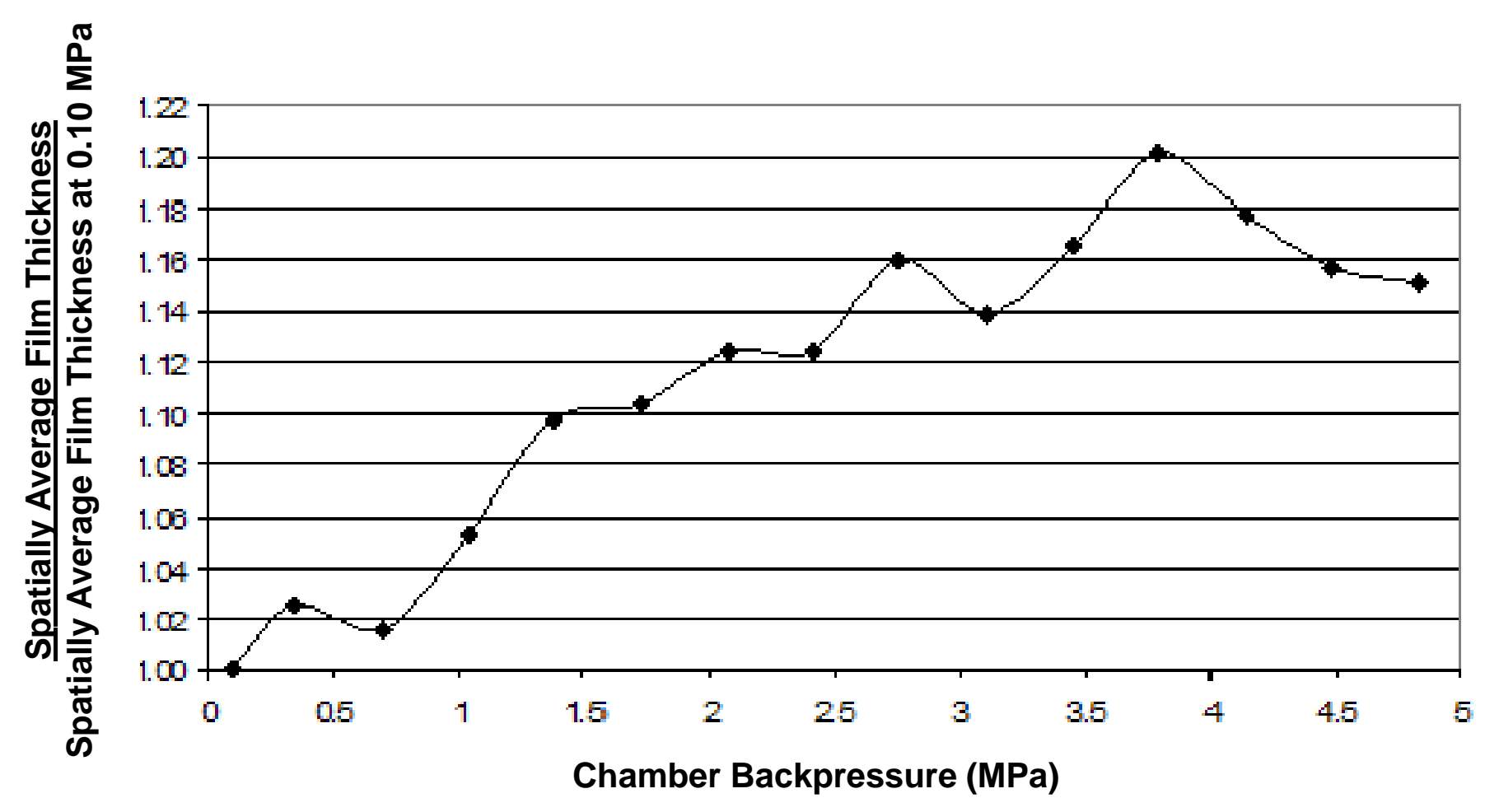

- Presence of hydraulic jump in flow distorts film thickness and induces susceptibility of flow to disturbances.

- For the same mass flow rate, increases in film thickness will raise the discharge coefficient and lower the issuing spray angle 


\section{Injector Pressure Drop and Discharge Coefficient at $0.09 \mathrm{~kg} / \mathrm{s}$}

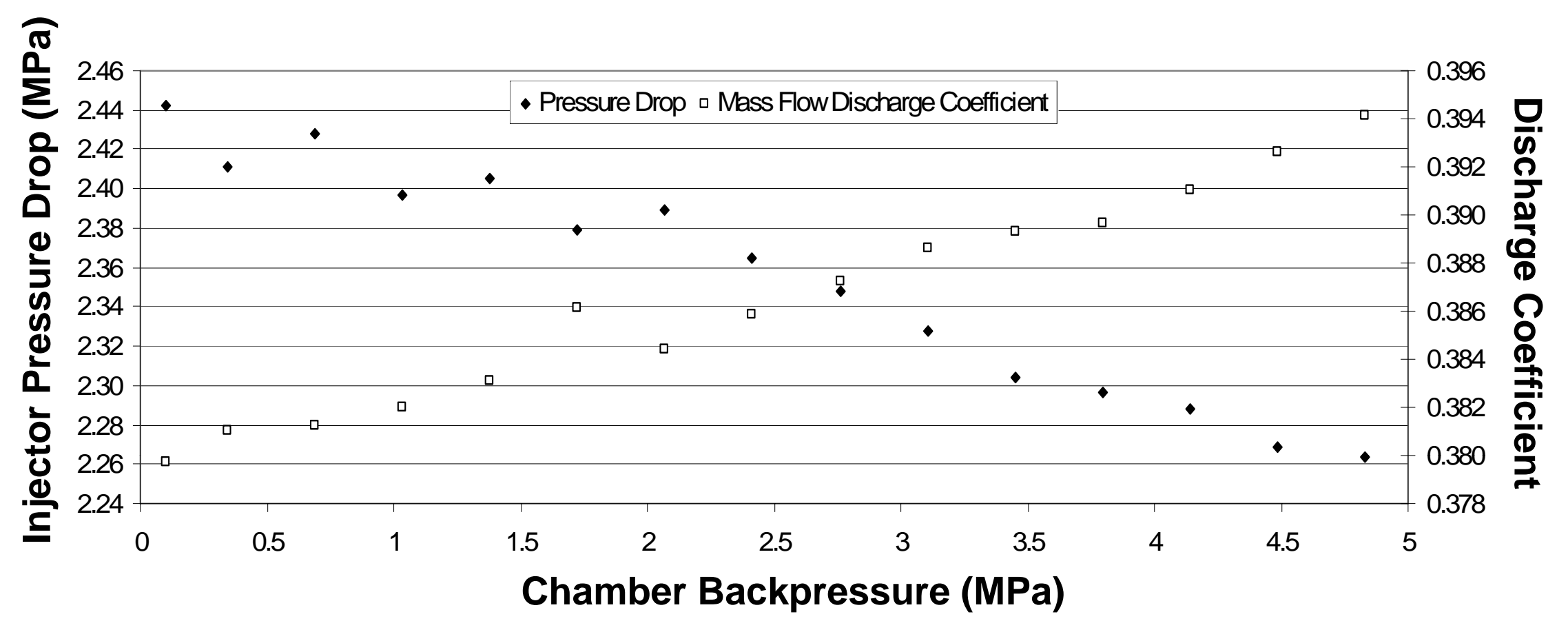

$$
C_{d}=\frac{\dot{m}_{\text {actual }}}{\dot{m}_{\text {ideal }}}=\frac{\dot{m}_{l}}{\rho_{l} V_{\Sigma} \frac{\pi}{4} D_{n}^{2}}=\frac{\dot{m}_{l}}{\sqrt{2 \rho_{l} \Delta P} \frac{\pi}{4} D_{n}^{2}}
$$




\section{Spray Angle Measurement}

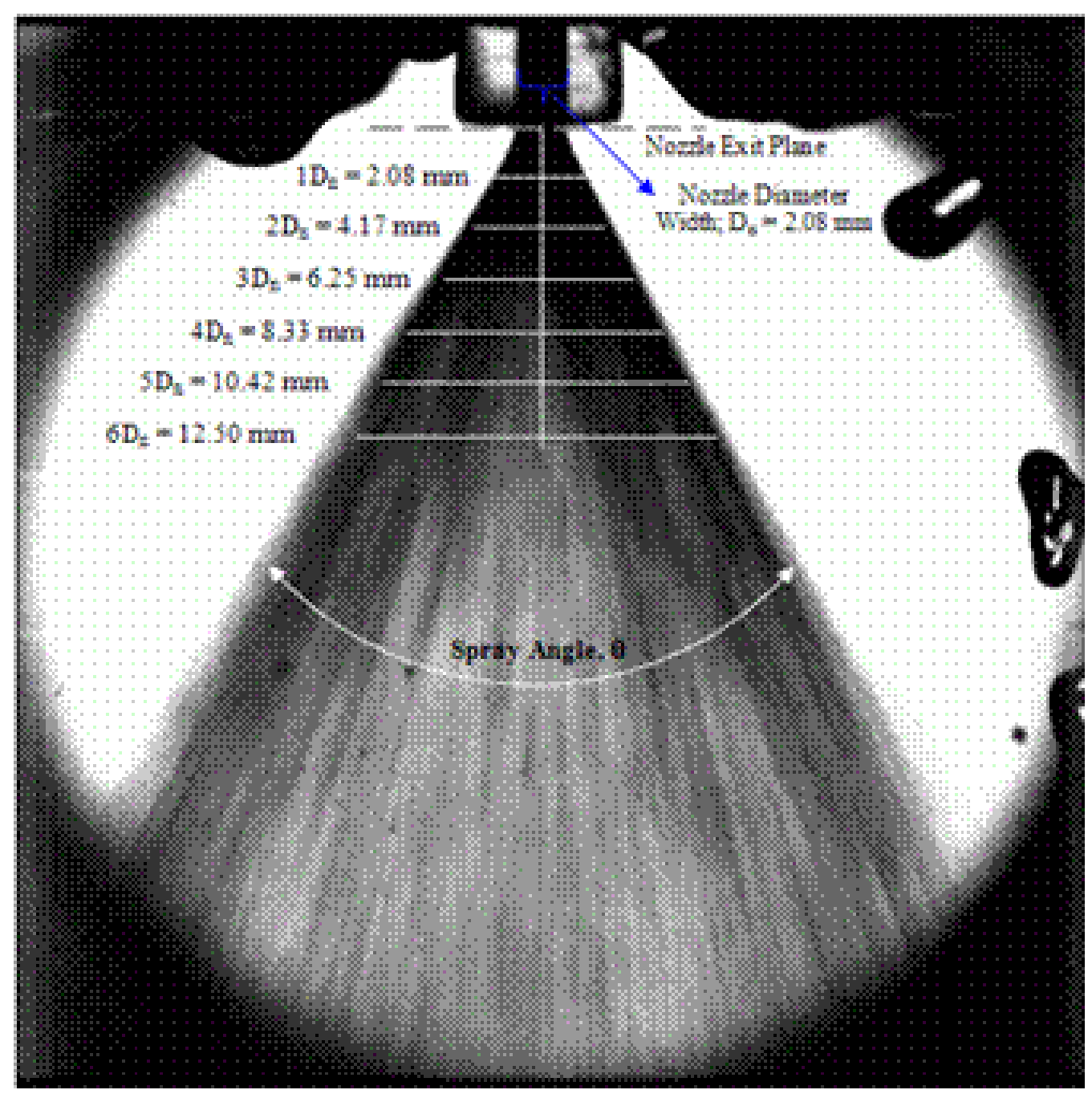

J. $\int_{\text {ESTS Group }}$ BCOS 


\section{Spray Angle Videography}

- Show movies of spray angle at full flow rate and chamber pressure of:

- $0.10 \mathrm{MPa}$

- $2.31 \mathrm{MPa}$

- $4.83 \mathrm{MPa}$ 


\section{Results: Spray Angle}

0.10 MPa: Pre-processed

0.10 MPa: Post-processed
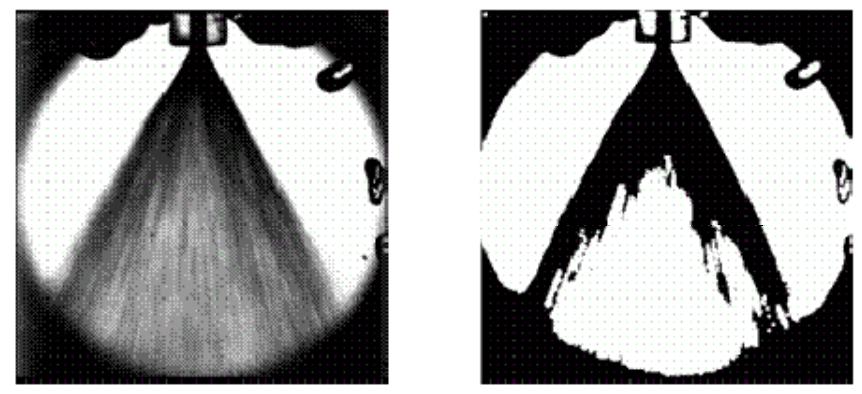

4.83 MPa: Pre-processed

4.83 MPa: Post-processed
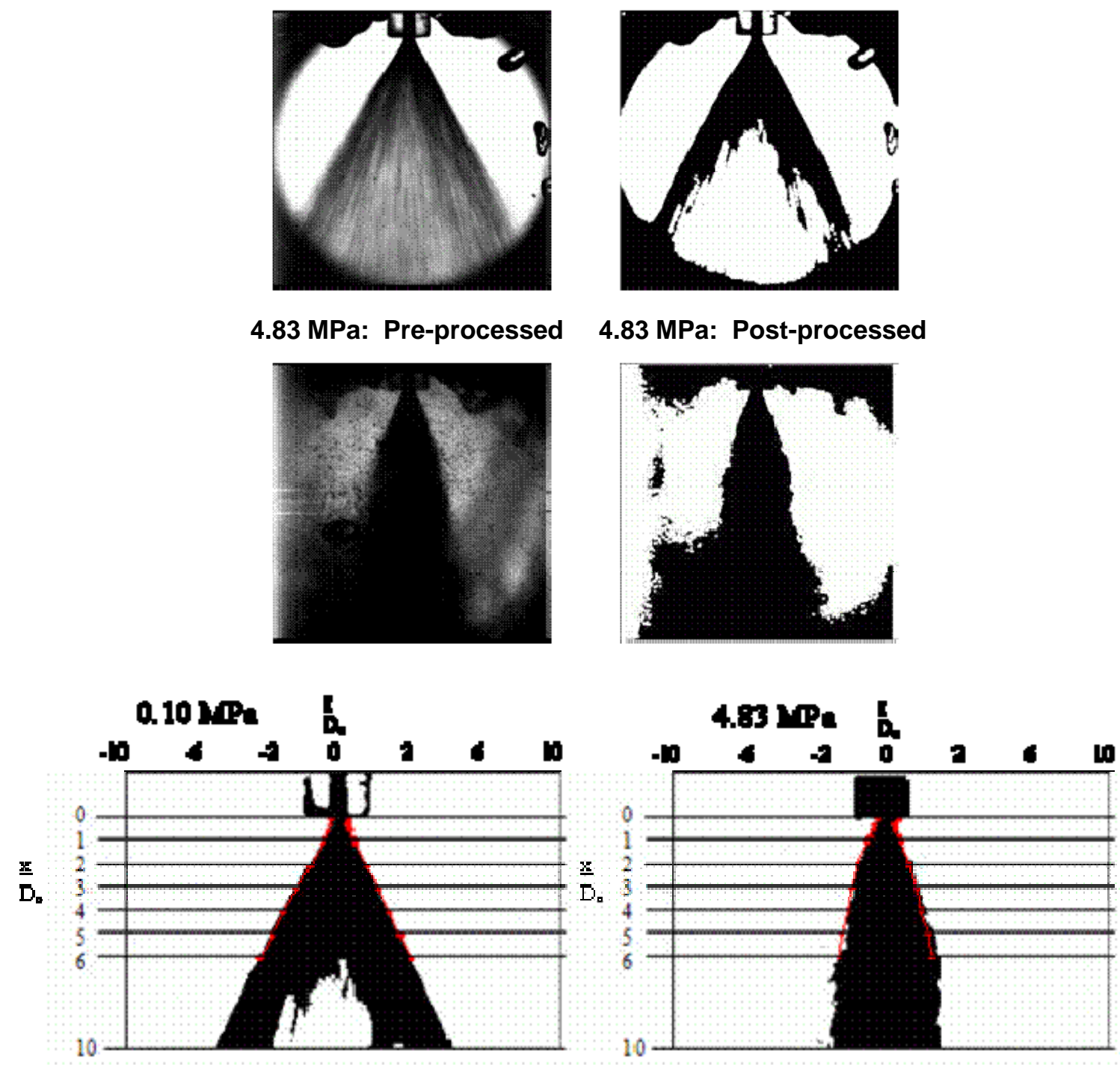


\section{Downstream Spray Angle}

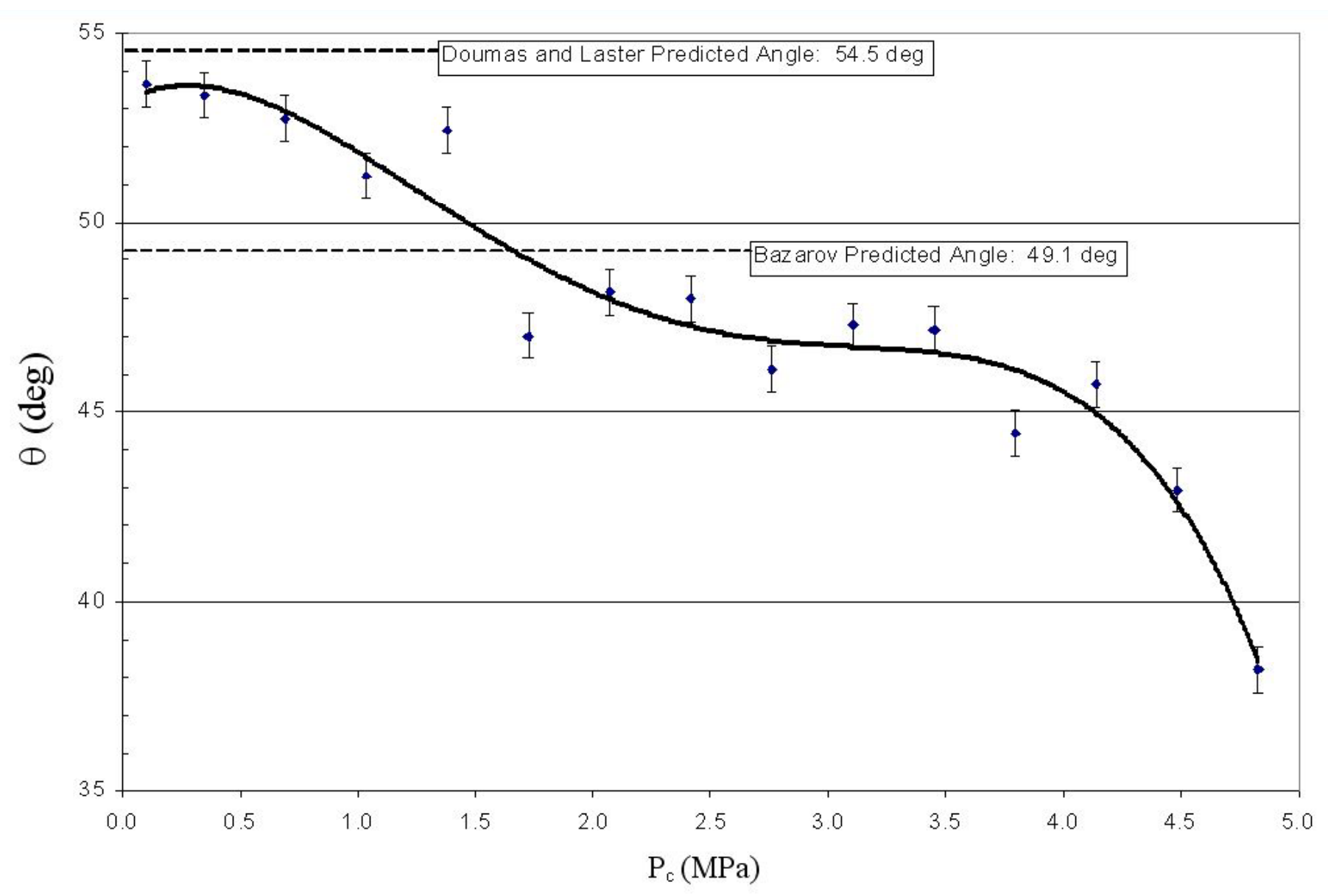

- Increasing chamber pressure causes more gas entrainment into the spray cone volume; angle decreases at a decreasing rate. 


\section{Conclusions}

- Film Thickness

- No numerical/analytical works exist that elaborate on hydraulic jump presence in liquid swirl injector

- Previous work exist on vortex breakdown in swirling flows at ambient conditions

- Sarpkaya: Adverse pressure gradient on vortex will cause pressure recovery and induce hydraulic jump

- Generally, increasing downstream pressure will induce and move jump upstream

- Increasing mass flow rate will cause similar effects

- Discharge Coefficient

- Increasing chamber backpressure raises discharge coefficient for particular mass flow rate operating range.

- Indicative of increasing viscous losses within swirl injector.

- Increased gas/liquid interface shear

- Increased axial flow retardation/recirculation within liquid annulus

- Spray Angle

- Increasing ambient pressure will lower design spray angles

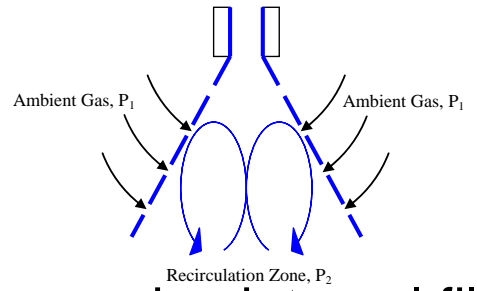

- Is correlated to the increasing internal film thickness of the nozzle, but not necessarily directly related. 


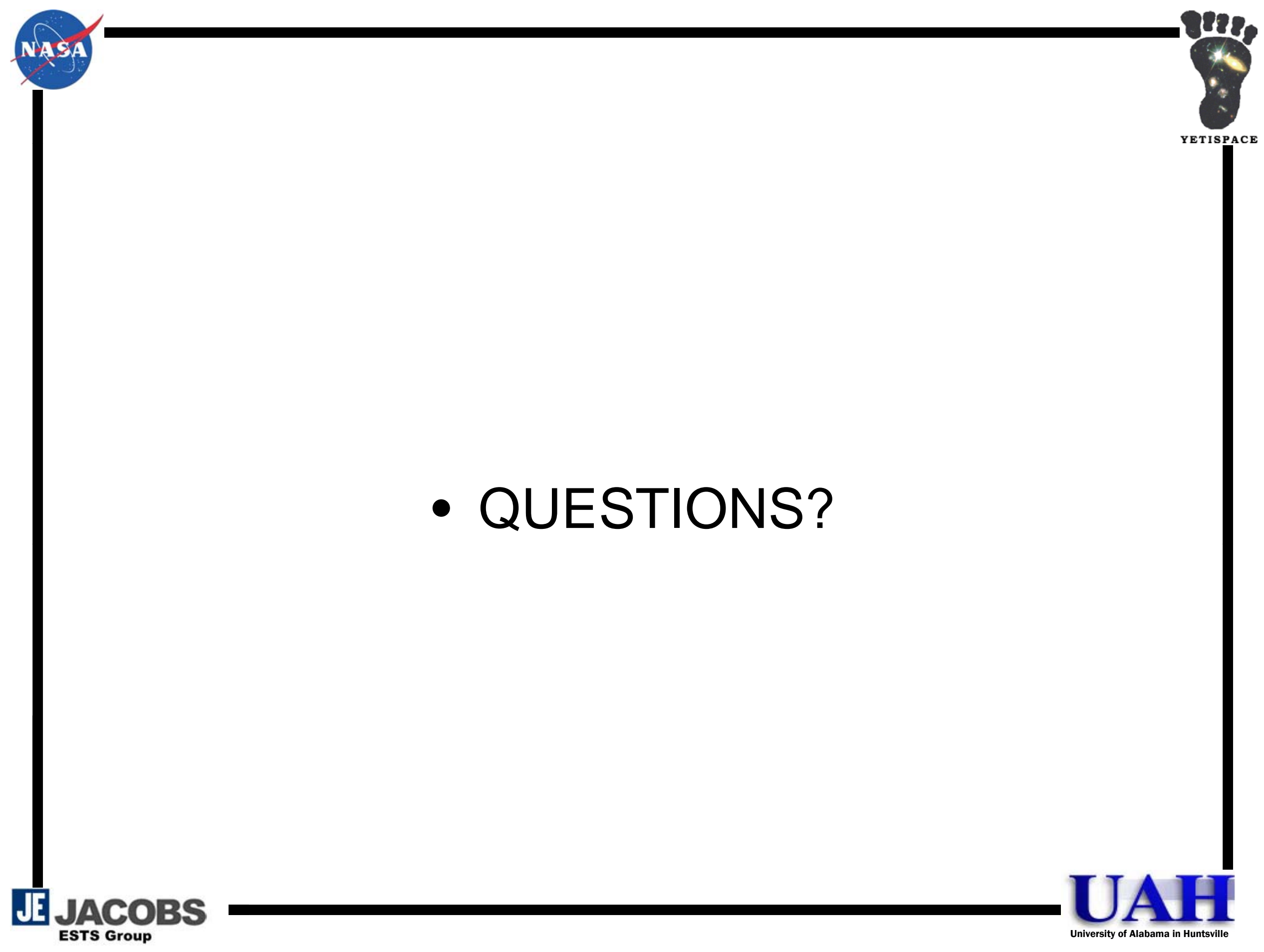




\section{Facility \& Hardware: Swirl Element Atmospheric Operation}

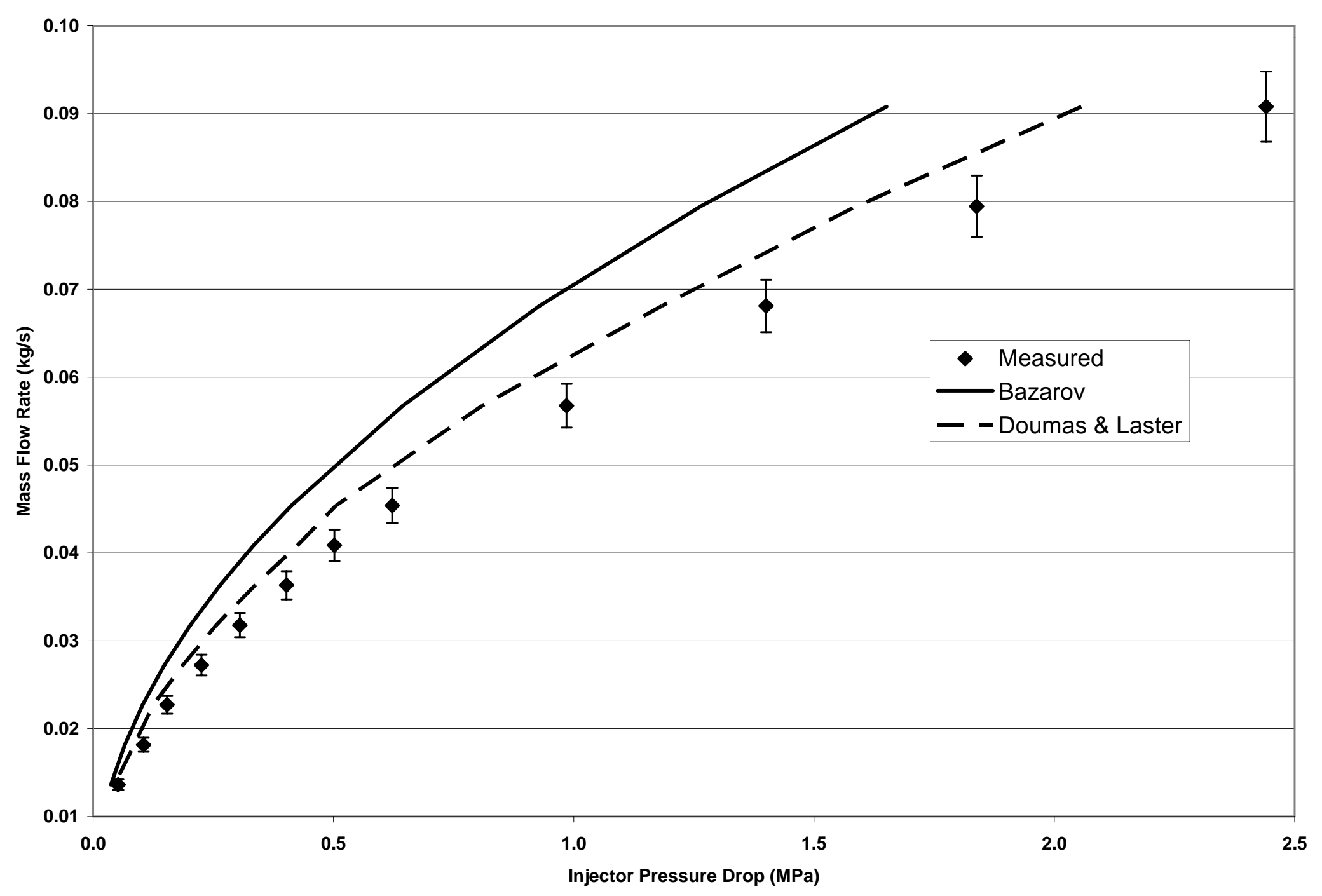




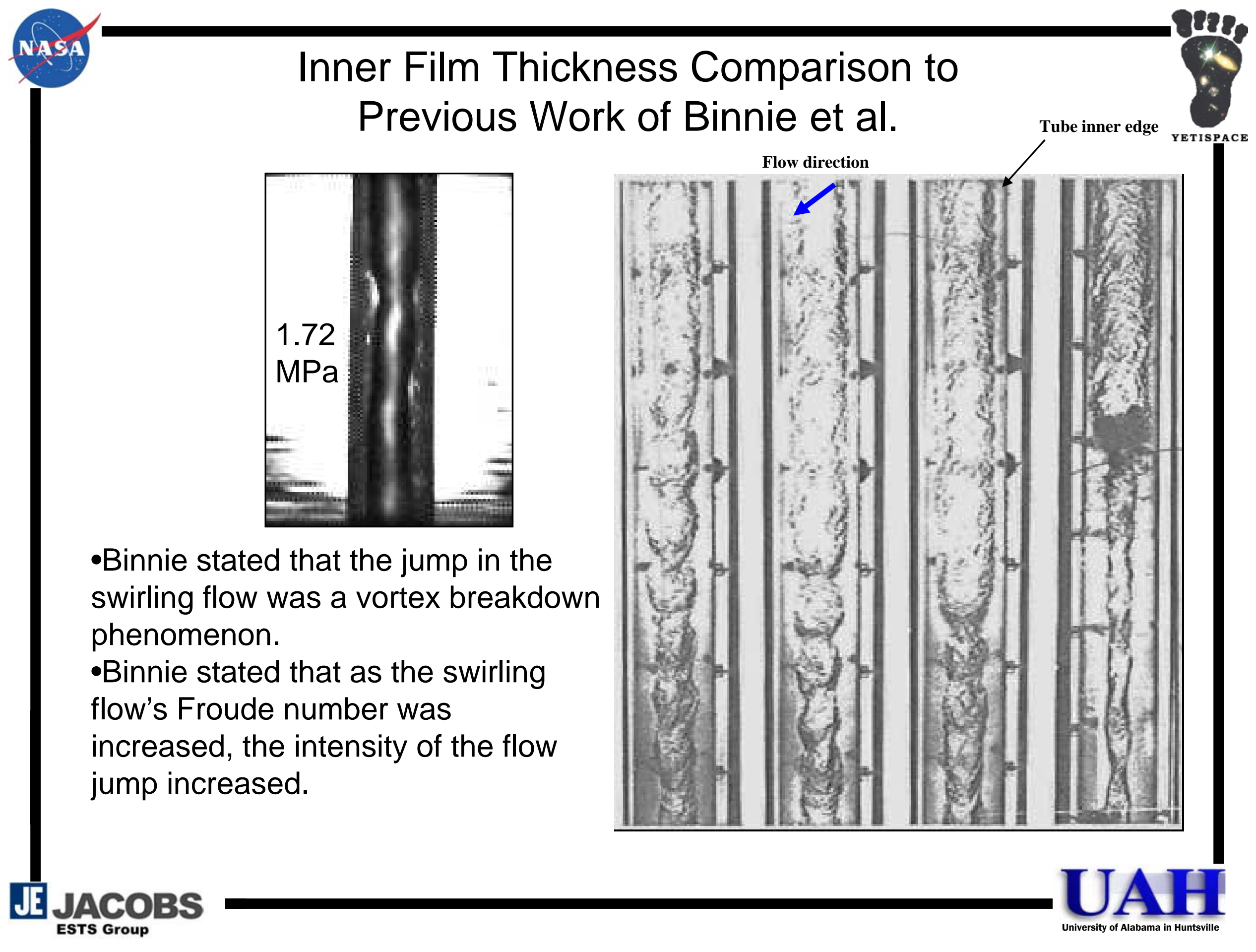




\section{Inner Film Thickness Vortex Breakdown}

Flow at $0.091 \mathrm{~kg} / \mathrm{s}$ and $0.10 \mathrm{MPa}$

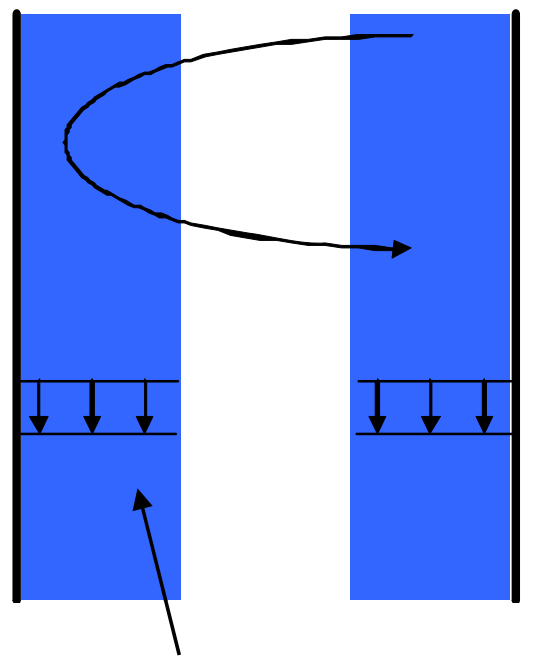

Mean axial velocity
Flow at $0.091 \mathrm{~kg} / \mathrm{s}$ and $\sim 1-1.4 \mathrm{MPa}$

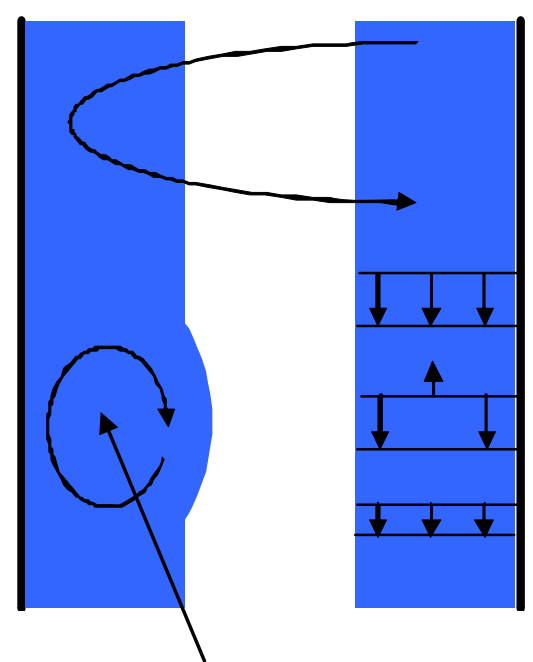

Unsteady Vortex Breakdown
Flow at $0.091 \mathrm{~kg} / \mathrm{s}$ and $>1.5 \mathrm{MPa}$

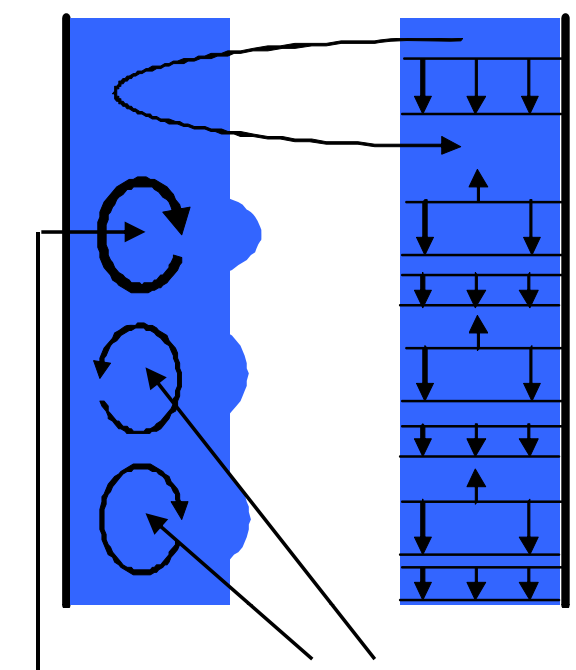

Parent and Child Vortex

Breakdowns

-Chamber pressure increase $->$ gas density in gas core increase -> increased shear -Axial flow retardation and flow recirculation $=>$ Vortex Breakdown -Vortex breakdown will move upstream into vortex chamber with increased momentum losses. 


\section{Inner Film Thickness Quantification}

High resolution digital stills were used to capture film thickness profiles

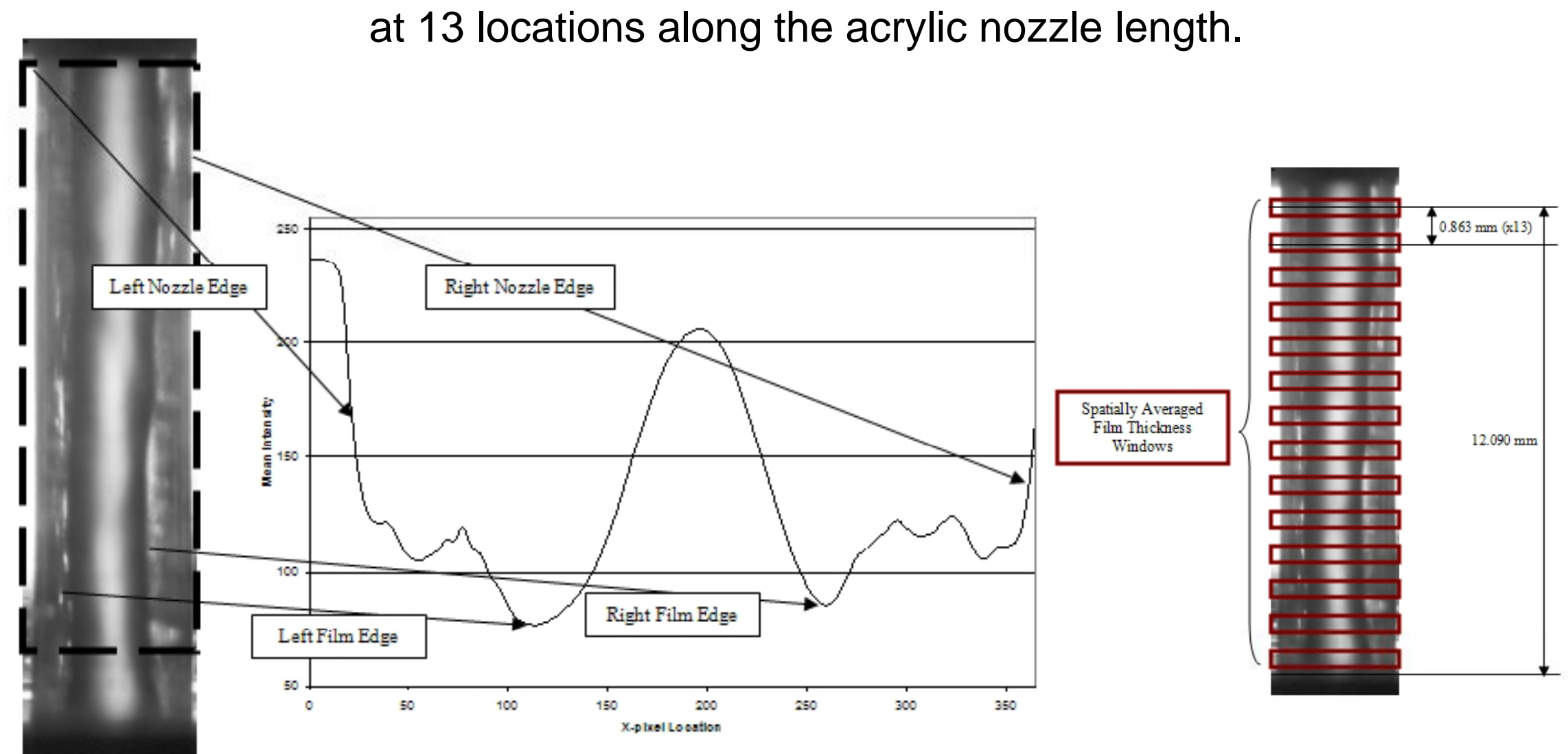

